\title{
Electricity Markets Ontology to support MASCEM's simulations
}

\author{
Gabriel Santos ${ }^{1}$, Tiago Pinto ${ }^{1}$, Zita Vale ${ }^{1}$, Isabel Praça ${ }^{1}$, Hugo Morais ${ }^{2}$ \\ ${ }^{1}$ GECAD - Research Group on Intelligent Engineering and Computing for Advanced Innova- \\ tion and Development, Institute of Engineering, Polytechnic of Porto (ISEP/IPP), Portugal \\ \{gajls, tmcfp, zav, icp\}@isep.ipp.pt \\ ${ }^{2}$ AUTomation and Control Group - Department of Electrical Engineering, Technical Univer- \\ sity of Denmark (DTU), Elektrovej, Building 326, DK-2800 Kgs. Lyngby, Denmark \\ moraiselektro.dtu.dk
}

\begin{abstract}
Power systems worldwide are complex and challenging environments. The increasing necessity for an adequate integration of renewable energy sources is resulting in a rising complexity in power systems operation. Multi-agent based simulation platforms have proven to be a good option to study the several issues related to these systems, including the involved players that act in this domain. To take better advantage of these systems, their integration is mandatory. The main contribution of this paper is the development of the Electricity Markets Ontology, which integrates the essential concepts necessary to interpret all the available information related to electricity markets, while enabling an easier cooperation and adequate communication between related systems. Additionally, the concepts and rules defined by this ontology can be extended and complemented according to the needs of other simulation and real systems in this area. Each system's particular ontology must import the proposed ontology, thus enabling the effective interoperability between independent systems.

Keywords: Electricity Markets, Multi-Agent Simulation, Ontologies
\end{abstract}

\section{Introduction}

The power sector business has been completely revolutionized by the emergence of liberalized Electricity Markets (EM). The sector's restructuring process brought out several challenges, requiring the transformation of the conceptual models that previously dominated the power sector [1]. This restructuring made the market more competitive, but also more complex, posing new challenges to its participants. Therefore, the involved entities are forced to rethink their behavior and market strategies.

To give entities decision support to address the new challenges, the use of simulation tools becomes decisive in order to study, analyze, and test different alternatives for

The present work was done and funded in the scope of the following projects: H2020 DREAMGO Project (Marie Sklodowska-Curie grant agreement No 641794); EUREKA - ITEA2 Project SEAS with project number 12004; AVIGAE Project (P2020 - 3401); and UID/EEA/00760/2013 funded by FEDER Funds through COMPETE program and by National Funds through FCT. 
markets' structure and evolution. For market participants it is important to anticipate scenarios and define strategies, while for the operators it is essential to test new market architectures. The main purpose of these tools is to deal with the constantly evolving reality of EM and grant actors with appropriate solutions to adapt themselves to the new reality, gaining experience to act in the context of a changing economic, financial, and regulatory environment. Market players aim to optimize their results (minimize costs if buying; or maximize profits if selling) and operators must ensure a competitive and transparent market in which no entity has significant market power [2].

EM simulators must be flexible in order to handle this complex and evolving reality, providing players with proper tools to adapt themselves to this dynamic reality and learn from experience. Several studies sustain that multi-agent systems (MAS) with the adequate simulation capabilities are suitable for the simulation of EM, considering the complex interactions of the involved players [3-5]. Some of the main advantages that multi-agent approaches provide are the facilitated inclusion of new models, market mechanisms, player types, and different types of interactions [6]. In this domain some reference modelling tools have emerged, such as AMES (Agent-based Modelling of Electricity Systems) [4], EMCAS (Electricity Market Complex Adaptive System) [3] and MASCEM (Multi-Agent Simulator of Competitive Electricity Markets) [6], [7].

MASCEM [6-8] is a modeling and simulation tool developed for studying complex restructured EM. It provides players with competitive advantage in the market by supplying them with simulation and decision-support resources.

Current tools are directed to the study of different EM mechanisms and to the analysis of the relationships between market entities, but they do not enable the interoperability with external systems. Simulators could gain significant added value by sharing their knowledge and market models with other agent societies. Such tools would provide the means for an actual improvement in current EM studies and development.

This paper introduces the Electricity Markets Ontology (EMO) designed to provide the means to achieve interoperability between EM simulation platforms. After this introductory section, an overview of MASCEM is presented in section 2. Section 3 presents the proposed EMO, while section 4 features a case study based on real data from several European EM operators. Finally, the conclusions are presented in section 5.

\section{MASCEM Overview}

MASCEM - Multi-Agent Simulator of Competitive Electricity Markets [6-9] is a modelling and simulation tool which has been developed aiming at studying the operation of complex and competitive restructured EM. It models the main complex and dynamic market entities and their interactions. To support players' decisions in accordance with their characteristics and goals, medium/long-term gathering of data and experience is also considered.

Software agents in MASCEM represent the main entities and players involved in EM, such as: market operator, system operator, buyer and seller agents (consumers, producers and/or prosumers), and the aggregators. Fig. 1 shows a general overview of MASCEM's multi-agent model. 


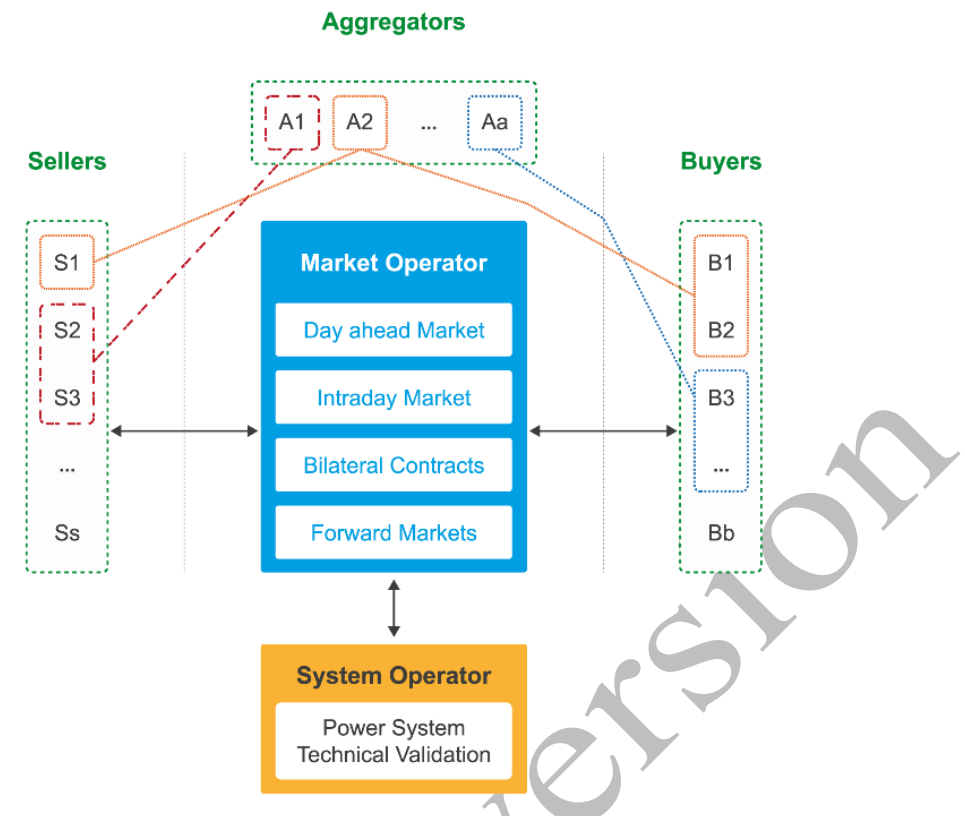

Fig. 1. MASCEM's multi-agent model, adapted from [6]

The market operator agent is responsible for coordinating and regulating the pool markets operation. It informs buyer and seller agents when the pool is open, receives their proposals, validates and analyses them, and determines the clearing market price, accepted and refused bids for each trading period.

The system operator agent is responsible for the system's security and ensures that all constraints are satisfied within the system. It is always present during the simulations. After being informed by the market operator of the market's outcome, it examines the technical feasibility from the power system point of view and solves congestion problems that may arise.

Buyer and seller agents - the market players - are the key elements of EM. Buyer agents represent the demand side entities, while generation units are represented by seller agents. On one hand, sellers compete with each other trying to increase their profits; on the other, they may also cooperate with buyer agents trying to reach agreements that are advantageous for both parties.

The aggregators represent alliances of small independent players. The meaningful increase of small independent producers and consumers participating in the market, brought the need to make such alliances to enable them competing with big producers. They manage their aggregates' information and are seen in the market as buyer or seller agents. Each aggregator is modelled as an independent MAS, allowing agents to be installed on different machines while maintaining the high performance as possible.

MASCEM includes the main types of negotiations normally present in EM, such as: day-ahead and intraday pool (symmetric or asymmetric, with or without complex conditions) markets; bilateral contracts and forward markets. By selecting a combination of these market models, it's also possible to perform hybrid simulations. 
The user defines each scenario by inputting the market and market type to simulate, the number of simulation days, the number of participating players and their strategies considering each type of agent, with their own decision-support resources, assuring them competitive advantage in the market.

Communications between agents are carried out through the exchange of messages. The Foundation for Intelligent Physical Agents (FIPA) suggests Agent Communication Language (ACL) as a standard for communications between agents [10]. Its content includes the content language, specifying the syntax, and the ontology which provides the semantics of the message assuring the correct interpretation [11]. MASCEM agents use the Electricity Markets Ontology (EMO) to enable the interoperability with other MAS or agent-based simulators that intend to participate in MASCEM's simulations.

\section{$3 \quad$ Electricity Markets Ontology}

Currently, MAS in the power system's domain are developed with their own specific ontologies. These systems share common concepts that are differently represented between the independently developed ontologies, and translating these concepts automatically is not as straightforward as it may seem. In order to take full advantage of the functionalities of those systems, there is a growing need for knowledge exchange between them.

FIPA suggests the use of an Ontology Agent (OA), which provides some related services, to solve the problem of multiple ontologies [12]. This is still an experimental standard and mappings between ontologies must still be performed by ontologies' designers, which increases the human effort required and costs of implementation. In alternative, Catterson [13] proposes the use of an upper ontology representing the general concepts of the domain, ensuring a common basis for the representation of those concepts and their relationships between systems while reducing the complexity of ontology mapping.

Inspired by this last approach, this paper proposed the use of EMO for the interoperability of EM multi-agent simulation platforms, which can be extended in a way to enable the full interoperability between those systems.

To reuse existing ontologies is usually a requirement for systems' interoperability. It is possible to find in the literature some ontologies developed for the field of energy markets, namely electricity and natural gas [14-16]. Unfortunately none is publicly available for reuse and/or extension, which led to the development from scratch of the proposed ontology.

In [14] a very extensive and interesting work has been developed, although the authors decided to take in mind only the domain of the ontology, leaving aside its application scenario. It is also important to note that the developed ontology is aimed at the Greek EM, not having been extended to any other European EM. If this ontology was publicly available, its reuse would be considered, given the extensity and accuracy of the knowledge already represented. In any case, ELMO ontology [14], as it is, is not suitable for EM multi-agent simulators. 


\subsection{EMO specification}

The EMO incorporates abstract concepts and axioms referring to the main existing EM. This ontology aims to be as inclusive as possible so that it can be extended and reused in the development of (lower level) market-specific ontologies, such as the MIBEL [17], EPEX [18], Nord Pool [19] or any other EM ontology. It was kept as simple as possible in order to facilitate its reuse and extension independently of the market's features and/or rules. However, given that the suggested ontologies were developed considering its use by agent based simulation tools, some markets' constraints were also defined in EMO. Fig. 2 illustrates EMO's relations between the identified classes and object and data properties.

From Fig. 2 it is possible to see the object properties represented in blue and the data properties defined within each class with the respective data types. The orange relations represent the inferred object properties, which are inverse properties of the ones defined in blue in the opposite direction.

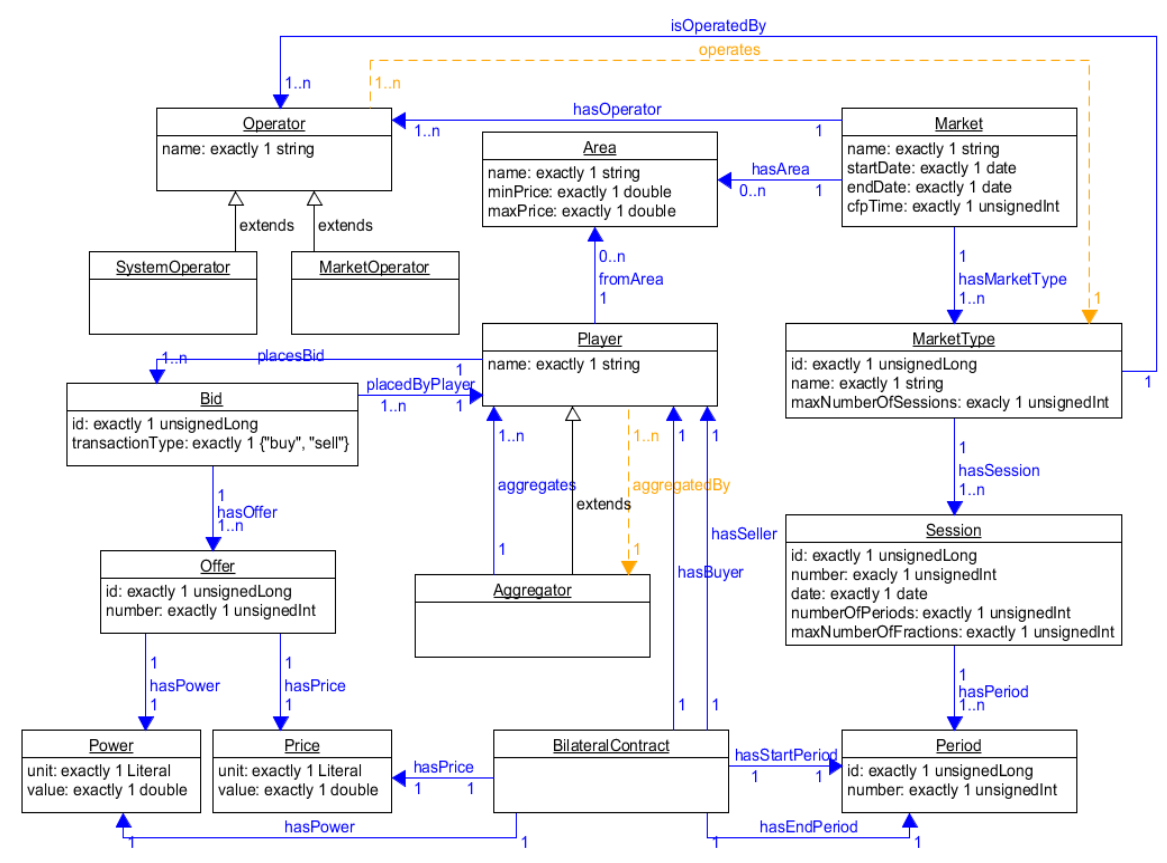

Fig. 2. Electricity Markets Ontology (EMO) ${ }^{2}$

It should be noticed that three object properties defined in this ontology are not present in the UML diagram, namely: hasBilateralContract, placedInPeriod and placedInSinglePeriod. These are important properties that are introduced in EMO to be reused by the ontologies defined by each EM's domain.

2 Available online: http://www.mascem.gecad.isep.ipp.pt/ontologies/imgs/fig2.png 
EMO has expressivity $A L C H I Q(D)$. The $A L$ (Attributive Language) is the base language allowing: (i) atomic negation, i.e. the negation of concept names that do not appear on the left side of axioms; (ii) concept intersection; (iii) universal restrictions; and (iv) limited existential quantification. $C$ is the Complex concept negation extension. The $H$ extension is related with the role Hierarchy (e.g. the sub properties). The $I$ extension represents the Inverse properties. The $Q$ extension are the Qualified cardinality restrictions, i.e. cardinality restrictions with fillers other than T. And finally, the $(D)$ refers to the use of datatype properties, data values or data types.

The definition of an Area includes a string name, a double minPrice and a double maxPrice. All the three data properties are defined as Functional. A functional property is a property that only relates the same subject to one single object/value. Each EM area has an identifying name and its minimum and maximum prices are usually defined in its market rules.

An Operator includes only a name, while the MarketOperator and SystemOperator classes are extended from Operator. Other types of operators may be present in different EM, which can be defined is each market's ontology after importing the EMO.

A Period is here identified only with an $i d$ and (period) number. These two properties are both Functional as well, and it has been found important to include them in this ontology due to simulation and data storage purposes. It is certain that a period (of time) can also be defined with a start and end instants, but that terminology was left open so that, if required, one can always extend its definition in the ontology by importing EMO.

Both Price and Power are defined as a set of a unit (e.g. EUR and MW respectively) and a value in double, being these two data properties Functional as well. An Offer, in turn, includes an id, a number and exactly a Power and a Price set by the object properties hasPower and hasPrice respectively. These two object properties are also Functional.

A Bid also includes an id, in addition to a transactionType ("buy" and "sell" only), a single Player (set with the Functional object property placedByPlayer) and Offers (set by the hasOffer object property).

A Player includes a name, and identifies its Area and placed Bids with the respective object properties fromArea and placesBid. The placesBid object property is the inverse of placedByPlayer, being also Inverse Functional, i.e. this property only relates the same object/value to a single subject. An Aggregator, on the other hand, is a subclass of Player, which aggregates other Players. The aggregates object property is inverse of the aggregatedBy object property, being this last inferred by the reasoner when active.

A Session includes an $i d$, a number, a date, the numberOfPeriods and the maxNumberOfFractions data properties, and also the Periods. The date data property is Functional, the numberOfPeriods identifies the number of periods to consider in the simulation, while the maxNumberOfFractions determines the maximum number of fractions (Offers) per Bid. The Periods are set with the hasPeriod object property, which is Inverse Functional.

The MarketType is defined by an id, a name, the maxNumberOfSessions, including its Sessions and Operators. The maxNumberOfSessions determines the maximum 
number of sessions to consider in the simulation. The Sessions and Operators are set with the hasSession and isOperatedBy object properties respectively. The hasSession property is Inverse Functional and the isOperatedBy is the inverse of operates object property, which is inferred by the reasoner.

A Market comprises a name, a startDate, an endDate, a cfpTime, and its Area(s), MarketType(s) and Operator(s). The startDate, endDate and cfpTime properties are Functional. The startDate and endDate describe the simulation start and end dates, from which are also determined the number of simulation days. The cfpTime sets the call for proposal time limit a MarketOperator will wait to receive the players' proposals. The Area(s) are set through the hasArea property, the MarketType(s) by the hasMarketType property and the Operator(s) via the hasOperafor object property.

A BilateralContract includes a buyer and a seller Player, a start and an end Period, a Power amount and a Price offer. The players are set by the hasBuyer and hasSeller Functional object properties. The start and end periods by the hasStartPeriod and hasEndPeriod properties respectively, where both are also Functional. And the hasPower and hasPrice properties set the Power and Price respectively.

Finally, the Area, the Operator, the Period, the Power, the Price, the Offer, the Player, the Bid, the Session, the Market, the MarketType and the BilateralContract classes are all Disjoint Classes, meaning that none of these classes has members in common. In other words, an element cannot be an instance of more than one of these classes, or else it makes the ontology inconsistent.

EMO was formulated in OWL DL, using Protégé $e^{3}$ tool, and its representation is in $\mathrm{RDF} / \mathrm{XML}$. It is publicly available ${ }^{4}$ so it can be used by third-party developers who wish to integrate their agent-based simulators with MASCEM, taking advantage of its simulation capabilities and market models. On the other hand, EMO may also be reused and extended for the development of new multi-agent simulation tools in the context of wholesale EM.

\subsection{Additional modules}

To enable semantic communication between the market operator and player agents, two additional modules have been developed separately from EMO. These are: (i) the Call For Proposal Ontology (CFP) and (ii) the Electricity Markets Results Ontology (EMR). Although these are not detailed in this paper, are also publicly available ${ }^{5}$.

The CFP has the purpose of being used by the market operator agents to ask player agents for bids to be placed in the market, and for players to send their proposals to the respective market operators. In turn, the EMR is the ontology used by market operator agents to inform player agents about their results and outcomes in the market.

Both modules have expressivity $A L C H I Q(D)$, similarly to EMO, and have also been formulated in OWL DL, being represented in RDF/XML as well.

\footnotetext{
http://protege.stanford.edu/

4 http://www.mascem.gecad.isep.ipp.pt/ontologies/electricity-markets.owl

5 http://www.mascem.gecad.isep.ipp.pt/ontologies/call-for-proposal.owl,

http://www.mascem.gecad.isep.ipp.pt/ontologies/electricity-markets-results.owl
} 


\section{$4 \quad$ Case Study}

This case study intends to demonstrate the usefulness and advantage of using EMO to support players' participation in the market. The simulation scenario was created with the intention of representing the European reality through a summarized group of players, representing buyer and seller entities of each area of each regional market. It includes two agents (buyer and seller) per area, practicing the average prices and negotiating the total amount of power that have been transacted in each of these areas in the reality, for the day $16^{\text {th }}$ January, 2013 (Wednesday).

Forty one areas are considered, i.e. 41 buyers and 41 sellers, resulting in a total of 82 players for this simulation. The selected market type is the symmetrical day-ahead pool without considering any complex offer or condition.

As the simulation starts, the market operator sends a call for proposal (CfP) to each registered player. Fig. 3 presents a snippet of the CfP sent by the market operator. The full version can be found online ${ }^{6}$.

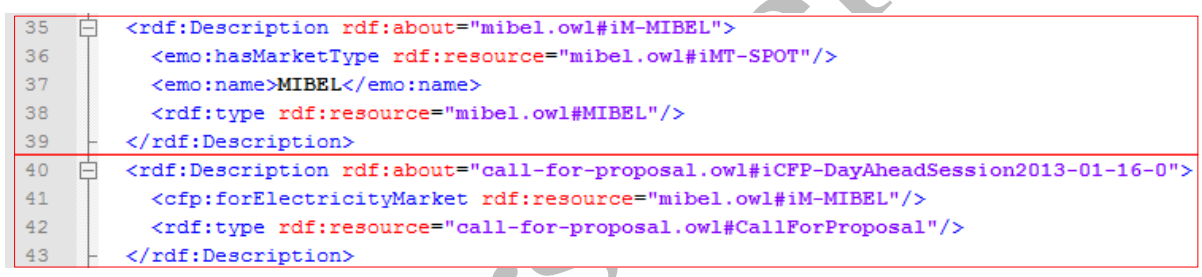

Fig. 3. CfP RDF snippet

Analyzing Fig. 3, it is possible to observe the definition of a CallForProposal (from line 40 to line 43) for the EM named "MIBEL" (defined from line 35 to 39).

After receiving the CfP, each player queries its knowledge base in order to send its proposal to the respective market operator. Fig. 4 presents a snip of the Proposal sent by agent Seller 38. The complete version is available online ${ }^{7}$.

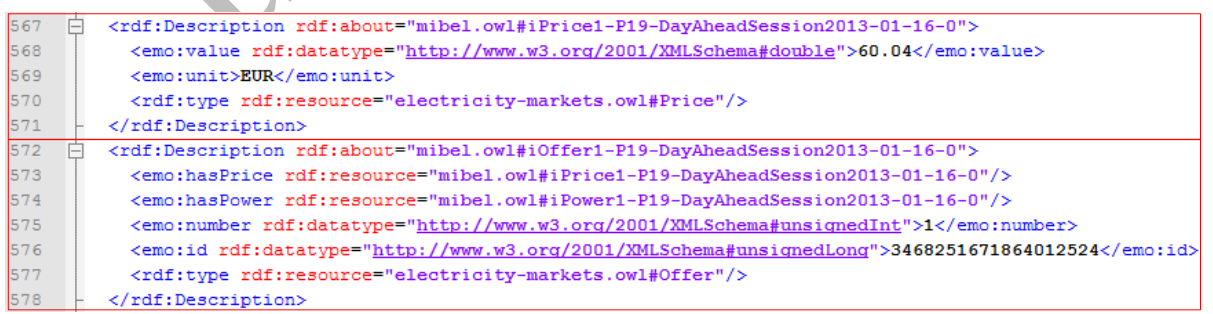

Fig. 4. Seller 38's Proposal RDF snippet

Observing Fig. 4, it is noticeable the definition of an Offer for period 19 (between lines 572 and 578). The Price proposed for this offer is defined from line 567 to 571.

6 http://www.mascem.gecad.isep.ipp.pt/ontologies/paper/paams/16/CfP.rdf

7 http://www.mascem.gecad.isep.ipp.pt/ontologies/paper/paams/16/Proposal.rdf 
After receiving the proposals and validating all incoming offers, the market operator analyses the bids, and generates the RDF results to be sent to the participating players. An excerpt of the RDF result achieved by Seller 38 is illustrated in Fig. 5. The full version of this RDF can be found online ${ }^{8}$, where the results may be observed with better insight.

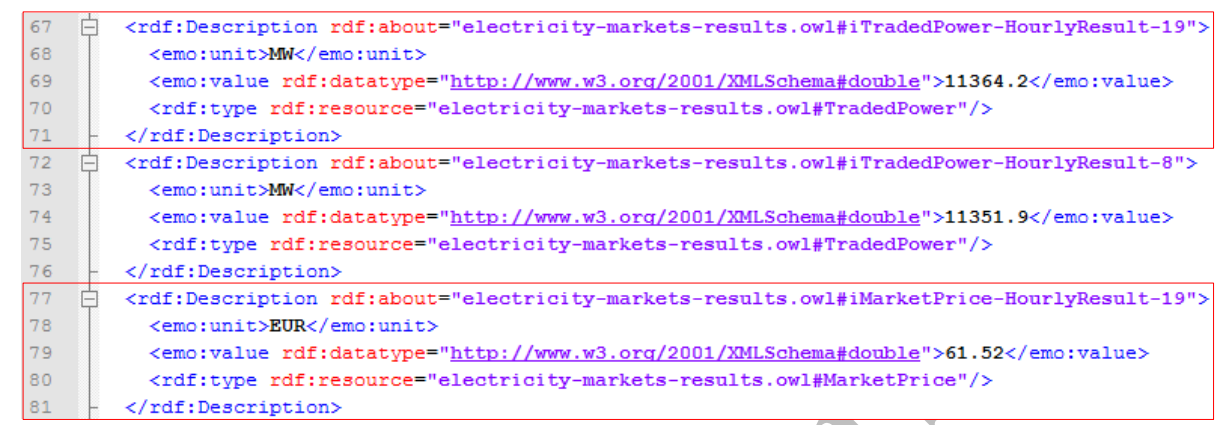

Fig. 5. Seller 38's Result RDF snippet

By the analysis of Fig. 5 it is possible to observe the traded power of Seller 38 (between lines 67 and 71) and market clearing price (from line 77 to line 81) of period 19.

Fig. 6 presents the results achieved by Seller 38 for each hourly period of the considered day.

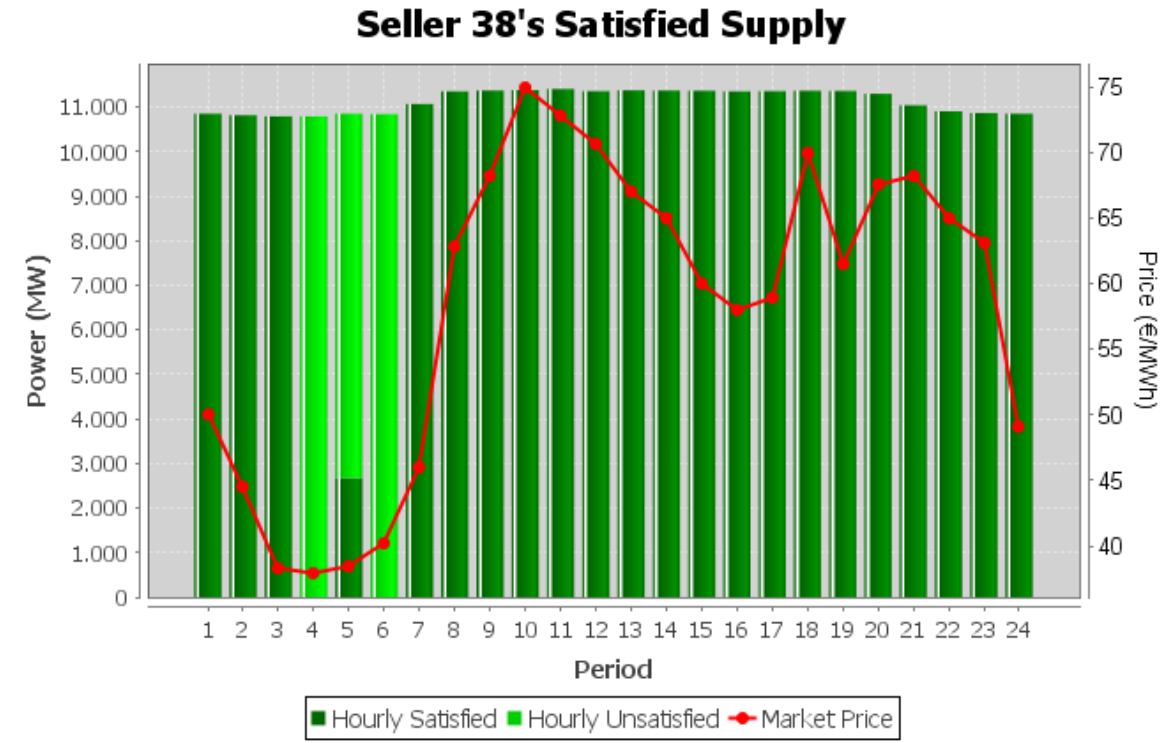

Fig. 7. Seller 38's satisfied supply

8 http://www.mascem.gecad.isep.ipp.pt/ontologies/paper/paams/16/Result.rdf 
As it is possible to notice, Seller 38 sold almost all its available power for the 24 hourly periods of the day. In periods 4 and 6 Seller 38 was not able to sell any of the offered power. In turn, in period 5, this agent is the one who determines the market price, being only able to sell less than $1 / 4$ of its available power.

It is also possible to observe that the market prices vary approximately between $€ 38$ (period 4) and €75 (period 10).

\section{Conclusions}

In order to disseminate the development of interoperable MAS within power engineering, interconnection issues must be addressed. To take full advantage of these systems, there is a growing need for knowledge exchange with the aim at providing full interoperability between different systems. With the objective of overcoming these issues, the Electricity Markets Ontology (EMO) is proposed, gathering the EM main concepts, enabling the interoperability of independently developed multi-agent based simulation platforms.

Additionally, particular modules conceived to deal with the different communications between market operators and market players have also been developed, namely: (i) the Call For Proposal Ontology (CFP) and (ii) the Electricity Markets Results Ontology (EMR).

Using EMO, different types of agents are able to communicate with each other, understanding a common language, while providing the means for any agent from external systems to do the same, simply by importing the developed ontologies. By "speaking the same language", agents from different communities can understand each other and communicate efficiently, without the need for spending unnecessary computational resources and execution time (which is an essential issue in a simulation process) in translating messages.

The developed ontology is publicly available online so it can be easily accessed, reused and extended by Ontology Engineers or MAS developers in the scope of EM. This is a relevant contribution, not only to provide the participation in joint simulations with MASCEM, but also to give the basis for the development of other systems specific ontologies. The comparison of the system's performance with and without the use of ontologies is considered as future work, as well as the conversion of RDF messages to JSON-LD 9 in order to reduce the computational weight of the communications.

The presented case study has proven the usefulness and advantages of using the proposed ontology in the scope of the wholesale EM. The new EM simulator resulting from the integration of the proposed ontology in MASCEM provides a solid platform to study and explore the implications and consequences of new and already existing approaches in EM. Researchers of the power systems area consider tools with this type of capabilities essential in order to be prepared to deal with the constant changes in the EM environment. It is important to point out that it was used real EM players' data in the simulation, extracted from the market operator's website.

\footnotetext{
${ }^{9}$ http://json-ld.org/
} 


\section{References}

1. Sioshansi F. Evolution of Global Electricity Markets - New paradigms, new challenges, new approaches. Academic Press, 2013.

2. Shahidehpour M., Yamin H. and Li Z. Market Operations in Electric Power Systems: Forecasting, Scheduling, and Risk Management. Wiley-IEEE Press, pp. 233-274, 2002.

3. Koritarov V. Real-World Market Representation with Agents: Modeling the Electricity Market as a Complex Adaptive System with an Agent-Based Approach. IEEE Power \& Energy magazine, pp. 39-46, 2004.

4. Li H. and Tesfatsion L. Development of Open Source Software for Power Market Research: The AMES Test Bed. Journal of Energy Markets, vol. 2, no. 2, pp. 111-128, 2009.

5. Migliavacca G. SREMS: a short-medium run electricity market simulator based on game theory and incorporating network constraints. IEEE Power Tech, Lausanne, Swiss, 2007.

6. Santos G. et al. Multi-Agent Simulation of Competitive Electricity Markets: Autonomous systems cooperation for European Market modelling. Energy Conversion and Management, 99, 387-399, July 2015.

7. Praça I., Ramos C., Vale Z. and Cordeiro M. MASCEM: A Multi-Agent System that Simulates Competitive Electricity Markets. IEEE Intelligent Systems, vol. 18, No. 6, pp. 54-60, Special Issue on Agents and Markets, 2003.

8. Pinto T., Praça I., Vale Z., Morais H. and Sousa T. Strategic Bidding in Electricity Markets: An agent-based simulator with game theory for scenario analysis. Integrated ComputerAided Engineering, IOS Press, vol. 20, no. 4, pp. 335-346, September 2013.

9. Vale Z., Pinto T., Praça I. and Morais H. MASCEM - Electricity markets simulation with strategic players. IEEE Intelligent Systems, vol. 26, no. 2, pp. 54-60 Special Issue on AI in Power Systems and Energy Markets, 2011.

10. Foundation for Intelligent Physical Agents (FIPA), FIPA Agent Management Specification, 2004. [Online]. Available: http://fipa.org/specs/fipa00023/, accessed on January 2016.

11. Foundation for Intelligent Physical Agents (FIPA), ACL Message Structure Specification, 2002. [Online]. Available: http://www.fipa.org/specs/fipa00061/, accessed on January 2016.

12. Foundation for Intelligent Physical Agents (FIPA), FIPA Ontology Service Specification, 2001. [Online]. Available: http://www.fipa.org/specs/fipa00086/, accessed on January 2016.

13. Catterson V. et al. An upper ontology for power engineering applications. April 2010. [Online]. Available: http://sites.ieee.org/pes-mas/, accessed on January 2016.

14. Alexopoulos P., Kafentzis K., Zoumas C. ELMO: An Interoperability Ontology for the Electricity Market. Proceedings of the International Conference on e-Business, Milan, Italy, July 7-10, 2009.

15. Dam K. and Chapping E. Coupling agent-based models of natural gas and electricity markets. In Proceedings of the First International Workshop on Agent Technologies for Energy Systems (ATES 2010), pages 45-52, 11 May 2010.

16. Dam K. and Keirstead J. Re-use of an ontology for modelling urban energy systems. Proceedings of the 3rd International Conference on Infrastructure Systems and Services: Next Generation Infrastructure Systems for Eco-Cities (INFRA), Shenzhen, China, 11-13 November 2010.

17. MIBEL - Mercado Ibérico de Electricidade, 2016. [Online]. Homepage: http://www.mibel.com/, accessed on January 2016.

18. EPEXSPOT - European Power Exchange, 2016. [Online]. Homepage: https://www.epexspot.com/, accessed on January 2016.

19. Nord Pool Spot, 2016 [Online]. Homepage: http://www.nordpoolspot.com/, accessed on January 2016. 